Aus der Poliklinik für Harnkrankheiten von Prof. C. Posner und Dr. J. Cohn in Berlin.

\title{
Prostatasekret und Prostatitis. Ein Beitrag
} zur Entzündungsfrage. ${ }^{2}$ )

Von Dr. C. Posner, a. o. Professor an der Universität, und Dr. L. Rapoport, Assistenten der Poliklinik.

\section{I.}

Seit geraumer Zeit, insbesondere aber seit den beweiskräftigen Untersuchungen von Robin und Fürbringer. herrscht Uebereinstimmung in der Frage nach der Beschaffenheit des normalen Sekrets der menschlichen Prostata: allseitig wird es bezeichnet als eine „dünne Milch", die sich mikroskopisch als Aufschwemmung kleinster Kügelchen (Prostatakörner") in einem eiweifhaltigen Fluidum charakterisiert. Diese Kügelchen, aus einem fettartigen Körper, und zwar einem Lecithin bestehend (Fürbringer), bilden den eigentlich spezifischen Bestandteil; außerdem kommen noch vereinzelte Epithelien, gelegentlich auch Corpora amylacea zur Beobachtung; auch kann man die Entscheidung, ob man es mit Prostatasaft zu tun habe, durch Darstellung der sogenannten Spermakristalle sichern - immer wieder muß aber betont werden, daß die Lecithinkügelchen dem Sekret seinen bestimmten, diagnostisch verwertbaren Charakter aufprägen.

Nun ist schon vielen Beobachtern aufgefallen, dab bei Entzündungen gerade dieser spezifische Charakter durch Schwinden der Lecithinkörner vollständig verwischt werden kann. Dab dies bei der akuten Abszedierung der Fall, bei welcher überhaupt reiner Eiter sich findet, ist am Ende nicht wunderbar. Aber sehr merkwürdig ist das Verhalten bei der chronischen Entzündung, bei welcher das Sekret zwar von der Norm abweicht, aber doch keineswegs in so auffallender Weise: das Kriterium wird hier zunächst nur durch das Erscheinen von Leukocyten gegeben, die dem normalen Sekret so gut wie ganz fehlen. Das Abnehmen, bezw. Verschwinden

1) Der Sektionsbefund ist veröffentlicht in der "Statistik der Todesursachen, erstattet von Prof. De y cke" in: Rieder: Für die Türkei Bd. 2, S. 150. Jena, Verlag von Gustav Fischer, 1904.

2) Nach einem Vortrag im Verein für innere Medizin am 6. März 1905. 
der Lecithinkörnchen hat daher hohe diagnostische Bedeutung, sodab z. B. Casper hierauf eine Einteilung der chronischen Prostatitiden basiert hat und als schwerste Formen eben diejenigen anspricht, in welchen diese Kügelchen gänzlich fehlen.

Es entsteht die Frage: Wo sind die Kügelchen geblieben? Werden sie nicht mehr abgesondert, oder verschwinden sie nachträglich aus dem Sekret?

In dieser Fragestellung liegt bereits eine Vorfrage enthalten: Wo ist in der Norm der UrsprungderKügelchen zu suchen - wo und wie gehen sie überhaupt in den Drüsensaft über?

Diese Vorfrage ist leicht zu beantworten: Die mikroskopische Untersuchung der normalen Drüse mit Zuhilfenahme geeigneter Färbemittel muß darüber Auskunft geben. Wir haben uns an einer großen Zahl verschiedenartiger Prostaten, welche im Pathologischen Institut der Königlichen Charité mit gütiger Erlaubnis des Herrn Geheimrat $0 \mathrm{rth}$, für welche wir auch hier unseren Dank aussprechen, verarbeitet wurden, davon überzeugt, daß der Vorgang der Lecithinabsonderung durch die Drüsenepithelien sich genau so abspielt, wie dies für die Fettsekretion in der Mamma, aber auch in vielen anderen Drüsen (Talgdrüsen, Tränendrüsen, Speicheldrüsen auch Samenblasen etc.) durch zahlreiche neuere Arbeiten (Benda, Unna, Traina, Plato u. a.) sichergestellt ist. Sowohl an Osmiumpräparaten als auch noch schöner nach Formalinfixierung und Färbung der Gefrierschnitte mit dem durch L. Michaelis eingeführten Scharlachrot lieb sich in allen Drüsengängen erkennen, daß das Lecithin in den Epithelien auftritt, und zwar in den einzelnen Zellen reihenweise uin den Kern geordnet. Der Lecithingehalt ist in den einzelnen Drüsen, aber auch in den einzelnen Teilen der gleichen Drüse verschieden, was wahrscheinlich mit der wechselnden Ruhe oder Tätigkeit del' Zellen zusammenhängt. Von hier geht es, anscheinend ohne daß die Zelle selbst dabei zunächst zerfällt, in Tröpfchenform in das Drüsenlumen über - also eine wahre Sekretion, wobei wir die Frage, ob das Lecithin sich in der Drüsenzelle selbst bildet, ob es präformiert in sie hineintritt oder synthetisch von ihr hergestellt wird, hier nicht erörtern wollen. Bisher haben wir - mit Ausnahme kindlicher Drüsen - noch keine Prostata untersucht, in welcher nicht eine lebhafte Lecithinbildung im Drüsenepithel sich gefunden hätte, sodaß wir also den Vorgang mit Bestimmtheit dorthin verlegen und die (früher meist geltende) Annahme, als handle es sich um einen sekundären Zerfall abgestoßener Zellelemente, als irrig bezeichnen müssen.1)

Die gleichen Fäıbungen geben nun auch darüber Auskunft, wo die Körnchen sich im fertigen Sekret eigentlich aufhalten. Schon vielfach - in erster Linie wiederum von Fürbringer - ist betont, dal im entzündlichen Prostatasaft sehr gewöhnlich große Körnchenkugeln vorkommen, die z. B. der eben erwähnte Autor direkt mit "Colostrumkörperchen" verglich. Unterwirft man nun ein Sekret von chronischer Prostatitis der Scharlachrotfärbung, so bekommt man ungemein prägnante Bilder: es zeigt sich, daß ein großer Teil der Leukocyten rotgefärbte Körnchen enthält, und zwar sind in den gewöhnlichen, polynukleären Zellen meist einzelne Körner nachweisbar, während andere, ganz vollgestopfte, große Zellen sich als mononukleäre "Makrophagen" im Sinne von Metschnik off erweisen. Diese Färbungen gelingen leicht am mit Formalin fixierten Trockenpräparat; noch viel zweckmäßiger und einfacher ist es, dem frischen Sekrettropfen einen Tropfell der Scharlachrotlösung direkt zuzusetzen - die Färbung tritt dann ungemein rasch, wenn auch nicht überall ganz gleichmäßig, ein, man kann das Präparat sofort mit einem Deckglas bedecken und mit Oelimmersion untersuchen. Namentlich für die diagnostischen Zwecke möchten wir dieses der „vitalen Färbung" analoge Verfahren in erster Linie empfehlen.

Denn in der Tat scheint es uns für die Diagnose von nicht unerheblicher Bedeutung. Gewil ist in vielen Sekreten auf diese Weise ein Fettnachweis zu führen (z. B. im Mundspeichel); auch an den gewöhnlichen Eiterzellen einer akuten

1) Ueber diese histologischen Untersuchungen behalten wir uns eingehendere Mitteilungen am anderen Orte vor.
Gonorrhoe oder im Sediment einer eitrigen Cystitis gelingt es mitunter, in den Zellen hier und da verstreute Fettkörnchen zu färben. Aber einmal haben wir bisher in keinem Falle von Gonorrhoe im Eiter eine auch nur annähernd so starke Reaktion der multinukleären Leukocyten gefunden wie bei der Prostatitis; dann aber halten wir in der Tat die großen Mononukleären mit ihrer vollständigen Erfüllung mit rotgefärbten Körpern und Ballen - wenigstens soweit es sich um ein Produkt der tieferen Harnwege handelt - für pathognomonisch, für Prostatasekret. Es bedarf wohl noch weiterer Untelsuchungen, ob etwa bei manchen Formen von Nephritis Aehnliches vorkommt - bishel haben wir in allen Fällen, in denen im Harnsediment solche Zellen nachweisbar waren, den Schlub auf eine gleichzeitig bestehende chronische Prostatitis bestätigt gefunden.

$\mathrm{DaB}$ es sich in diesen Bildern um das gesuchte Lecithin handelt, ist wohl nicht zu bezweifeln, da wir im normalell Sekret die feinen Körnchen ganz in derselben Weise gefärbt gesehen haben. ${ }^{1}$ Freilich wollen wir gleich hier auf zwei Fehlerquellen aufmerksam machen, welche sich Nachuntersuchern eventuell störend bemerkbar machen könnten: Einmal darf nicht del. Untersuchung von Urethralsekret eine Bougierung oder Katheterisierung unter Anwendung von Vaseline kurz voraufgegangen sein, da es hiernach leicht zı Fettdepots kommt. Dann aber ist zu beachten, daß auch die Epithelien der Harnröhre - insbesondere wohl die der Drüsen - Fettreaktion geben; sie erscheinen wie mit kleinsten roten Punkten bestreut. Das Obengesagte ist ausschließlich auf die Leukocyten zu beziehen. Anschließend wollen wir noch hervorheben, daß Spermatozoen im Sekret, beziehungsweise in frischem Samen keine Fürbung mit Scharlachrot annehmen, eine Tatsache, die allerdings im Widerspruch mit einer Beobachtung Trainas steht, welcher angibt, er habe die Spermatozoenköpfe im Samenblaseninhalt gefärbt gesehen. Uebrigens nehmen diese auch bei Behandlung mit Osmiumsäure keine schwarze Färbung an.

Die bisher mitgeteilten Befunde lassen erkennen, wo bei chronisch entzündlichen Zuständen der Prostata das Lecithin bleibt: es wird von den weißen Blutzellen aufgenommen und muß in demselben Maße aus dem Sekret schwinden, wie die Zahl der Eiterzellen steigt - also eine Bestätigung der oben angezogenen praktischen Beobachtung Caspers. Es ist nicht ohne Wert, dies besonders zu betonen, weil man sonst leicht geneigt sein könnte, aus dein Fehlen des Lecithins auf primäre, tiefergreifende Erkrankung der lecithinproduzierenden Drüsenzellen selbst zu schließen.

\section{II.}

Haben wir es demnach hier mit einer echten Phagocytose zu tun, so entsteht die weitere Frage: Was veranlabt die Phagocyten zu ihrer Wanderung in das Drüsenlumen? Man ist gewöhnlich mit der Antwort auf diese Frage rasch bei der Hand: Die Schuld wird den pathogenen Mikroorganismen zugeschoben, die Auswanderung der weißen Blutkörperchen als eine Reaktion auf den durch sie gesetzten Reiz, als Wirkung der - um es mit modernem Schlagwort auszudrücken - "positiven Chemotaxis" der Bakterien angesprochen. Diese Anschauung ist $z$ weifellos für viele Fälle vollberechtigt - kein Mensch stellt heute eine solche Wirkung der mikrobischen Krankheitserreger in Frage. Auch für viele Fülle von chronischer Prostatitis mag sie gelten. Sehen wir sie doch oft genug im Anschlub an eine urethrale Infektion, besonders an die Gonorrhoe sich entwickeln - wenn auch keineswegs immer durch den Gonococcus selber, sondern durch eine Sekundäroder Mischinfektion bedingt, wie dies unter anderem die Untersuchungen von J. Cohll dargetan haben. Aber neben diesen zweifellos infektiösen Prostatitiden gibt es doch, darüber sind heute wohl alle Beobachter einig, auch solche, die sich ohne jode voraufgegangene Infektion entwickeln - aseptische Formen, wie sie seitens der Guy onschen Schule (Le Fur u. a.) genannt werden. Nicht nur vermissen wir sehr oft im jrosta-

1) Wir wollen nicht unterlassen, darauf aufmerksan zu machen, daß die freien Lecithinkōrner sich etwas langsamer und weniger intensiv zu färben pflegen als die in Zellen eingeschiossenen. 
tischen Sekret, mag es auch noch so leukocytenhaltig sein, jede Spur eines Mikroorganismus - wir können auch in einer ganzen Reihe von Fällen aus der Anamnese den bestimmten Nachweis führen, daß überhaupt niemals eine Infektion voraufgegangen ist. Gewiß ist in dieser Hinsicht die größte Skepsis angebracht; aber ich (Posner) habe allein im letzten Jahre in der Privatpraxis vier Fialle gesehen, welche, wie ich glaube, der strengsten Kritik standhalten. Jedesmal handelte es sich um Personen (ausschlieblich russische Juden), bei denen aus religiösen Gründen jeder infektiöse Coitus ausgeschlossen war, entweder um in früher Jugend Verheiratete oder sogar um solche, die überhaupt noch niemals den Beischlaf ausgeübt hatten. In diesen Fällen, die ja jedem Beobachter bekannt sind, konnten dann freilich meist andere Momente ermittelt werden, welche eine Schädigung des Genitalapparates bedingen - sexueller Abusus, Coitus interruptus, Masturbation. Neben diesen werden auch mechanische Ursachen - Radfahren, Reiten und dergleichen -- vielfach angeschuldigt. Im übrigen aber unterscheiden diese Formen sich in keiner Weise von den postgonorrhoischen - sie bedingen dieselben subjektiven und objektiven Erscheinungen und können, wie wir wiederholt gesehen haben, sogar auf die Beschaffenheit des Samens den gleichen deletären Einfluß (Nekrospermie) ausüben.

Lie Erklärung dieser Fälle begegnete bisher großen Schwierigkeiten. Man half sich wohl mit der Hypothese, daß die kongestionierte oder überangestrengte Drüse ein gutes Receptaculum für etwa zufällig eingewanderte Bakterien bildet, die unter günstigen Vorbedingungen leicht eine Entzündung auszulösen vermöchten - einen Beweis für diese Vermutung zu geben, war aber, mangels mikroskopischer Befunde, schwer.

Wie uns scheint, fällt nun auf diese Dinge ein neues und völlig aufklärendes licht, wenn man den oben schon gezogenen Vergleich mit Milch und Colostrum nochmals ins Auge faßt. ber Streit über die Natur der Colostrumkörperchen kann wohl. wenn auch hier und da noch dissentierende Stimmen laut werden (wie z. B. jüngst in einer Verhandlung der Wiener Gesellschaft für innere Medizin und Kinderkrankheiten), jetzt in dem Sinne als abgeschlossen betrachtet werden, daß die zuerst von Czerny aufgestellte Anschauung, es handle sich hier um eingewanderte und mit Milchfett erfüllte Leukocyten, zu Recht besteht; die Arbeiten von Benda, Unger u. a. sprechen unzweideutig in diesem Sinne. Ganz besonders aber kommt einer neuerlich aus der v. Leydenschen Klinik hervorgegangenen, unter der Leitung von L. Michaelis ausgeführten Arbeit von $\mathrm{H}$. Bab das Verdienst zu, über diese Frage Klarheit verbreitet zu haben. Bab hat nicht blok nochmals mit genauer Detailarbeit die Natur der bei der Colostrumbildung beteiligten weißen Zellen (Makro- und Mikrocyten) ermittelt, sondern auch den ursächlichen Zusammenhang und das Verhältnis des Vorganges zur Entzündung klal dargestellt. Nach seinen Ergebnissen müssen wir annehmen, daß eben das Milchfett selbst, wenn es in den Drüsengängen sich aufstaut - wie dies am Ende der Schwangerschaft und kurz nach der Entbindung der Fall - einen „positiv-chemotaktischen“ Reiz auf die Leukocyten ausübt, welche dann in die Drüsengänge einwandern und das aufgespeicherte Fett als Freßzellen in sich aufnehmen. Der Vorgang ist von dem einer "echten" Entzündung, welche durch Bakterien ausgelöst wird, höchstens quantitativ verschieden; wie sehr er sich in jeder Hinsicht einer wirklichen Entzündung nähern kann, wird namentlich durch die Fälle sogenannten Milchfiebers illustriert.

Was für die Milchdrüse gilt, scheint uns auch für die Prostata zuzutreffen. Auch hier fehlen im Sekret, solange der Abfluß ungehemmt ist, die Leukocyten, auch hier erscheinen sie sofort, wenn eine Stauung vorliegt, und bemächtigen sich des unverwertet daliegenden Materials - wahrscheinlich auch,. um es durch Rücktransport in die Lymphbahnen dem Organismus wieder nutzbar zu machen. Wir haben esalso mit einer reaktiven Entzündung zu tun, in der wir einen außerordentlich fein funktionierenden, automatischen Regulationsvorgang des Organismus erblicken dürfen. Wir bezweifeln keinen Augenblick, daß das hier zutage tretende Gesetz auch für andere Drüsen des Körpers Geltung hat; man- cherlei ältere Versuche - z. B. über die Folge der Unterbindung des Wirsungschen Ganges für das Pancreas - lassen sich ungezwungen in diesem Sinne deuten. Man wird nicht umhin können, diese Auffassung in ihrem allgemein pathologischen Werte weiter zu prüfen; vielleicht ergibt sich, daß neben den in letzter Zeit etwas einseitig betonten Entzündungen bakteriellen und toxischen Ursprungs gerade diesen Retentionsentzündungen eine große Bedeutung zukommt.

Für die praktische Beurteilung unseres Spezialfalles der chronischen Prostatitis ergibt sich hieraus zweierlei: Zunächst mub man bei der Diagnose noch mehr als bisher zu unterscheiden suchen, ob man es mit infektiösen oder aseptischen Formen zu tun hat. Man versteht jetzt, warum alle oben erwähnten ätiologischen Momente eine Entzündung hervorrufen können: jede Kongestion, jede Erschlaffung des Muskeltonus der Drüse durch Erschöpfung kann Sekretstauung mit ihren skizzierten Folgen bedingen; freilich wird man auch in Zukunft dem Erscheinen vereinzelter Leukocyten im Sekret nicht mehr so viel Bedeutung beilegen dürfen, wie dies bisher geschehen war: die Uebergänge von einfacher Sekretverhaltung zur echten Entzündung vollziehen sich ziemlich unmerklich, und die Abgrenzung der chronischen Prostatitis, über deren Häufigkeit ja bisher schon eine auffällige Uneinigkeit unter den Beobachtern herrschte, wird immer schwieriger. Insbesondere aber wird man jetzt verstehen, warum so oft andersartige Erkrankungen der Prostata sich mit chronischer Entzündung kombinieren. Es kann uns nicht mehr wundernehmen, wenn wir z. B. bei beginnender oder ausgebildeter Hy pertrophie, die ja ohne Ansammlung von Sekret in den sich verengernden Drüsenrïumen kaum denkbar ist, so oft auch die Erscheinungen chronischer Entzündung antreffen; aber wir werden aus dieser Koinzidenz unseres Erachtens nicht mit Ciechanowski, Rothschild u. a. den Schluß ziehen dürfen, daß nun die chronische Entzündung den Anlab zur Hypertrophie gegeben habe, vielmehr umgekehrt in ihr das primäre Moment, in der Entzündung aber mit Albarran, Motz, Goldschmidt u. a. eine Komplikation oder einen Folgezustand erblicken!

Weiter aber werfen unsere obigen Ausführungen auch auf die Therapie ein neues Licht. Jedem, der viele Fälle von chronischer Prostatitis zu sehen Gelegenheit hat, ist die Tatsache geläufig, daß man mitunter ganz evidente und schnelle Erfolge mit den üblichen Maßnahmen erzielt - andere Male hingegen höchstens langsame Besserungen, aber keine Beseitigung des Krankheitsprozesses. Als wirksamste unter den therapeutischen Eingriffen gelten nun schon lange diejenigen, welche in mechanischer oder physikalischer Weise die Drüse beeinflussen; in erster Linie also Massage, Hydrotherapie, Elektrizität. Allen diesen ist gemeinsam, dab sie, wie ich (Posner) stets in meinen Vorlesungen betont habe, den Tonus der Muskulatur heben - eine Auffassung, der noch ganz neuerdings Anton Bumm in Wien ausdrücklich beigetreten ist; weit weniger die einmalige, brüske Entleerung der Drüse von ihrem Inhalt soll z. B. durch die Massage bezweckt werden, als vielmehr die allmähliche Kräftigung ihrer Detrusorfasern, die dann das sich stauende Sekret wieder auszutreiben befähigt werden. Die Bedeutung gerade dieses Faktors tritt nun in erhöhtem Maße hervor; und es begreift sich nun, warum wir die "atonischen" Formen (Pórosz) günstig beeinflussen können, während bei der echten Infektion der Drüse diese Versuche ebenso wie die Anwendung urethraler Therapie nur zu oft erfolglos blieben.

Wir fassen kurz zusammen: Das Lecithin wird in der Prostata, ebenso wie das Milchfett der Mamma, seitens der Epithelien sezerniert und dem Drüsenirhalt beigemischt; ist der Abfluß des Sekrets gehemmt, so erfolgt eine Leukocyteneinwanderung, und die Leukocyten nehmen das Lecithin durch Phagocytose auf; ein Teil der Fälle chronischer Prostatitis beruht einfach auf solcher Sekretstauung, und es sind das diejenigen Formen, welche einer mechanischon Therapie die günstigsten Aussichten bieten. 